\title{
Combinational Therapy with Aspirin and Ticagrelor Alleviates Vascular Inflammation and Angiotensin II-driven Abdominal Aortic Aneurysm Formation in Mice
}

\author{
Xiaowei Liu \\ Zhejiang Hospital \\ Yingzheng Weng \\ Zhejiang Hospital \\ Jiangjie Lou \\ Zhejiang Hospital \\ Xiaofeng Chen \\ Taizhou Hospital, Wenzhou Medical University \\ Changqing Du \\ Zhejiang Hospital \\ Lijiang Tang ( $\nabla$ zjyytang@126.com ) \\ Zhejiang Hospital
}

\section{Research Article}

Keywords: Abdominal aortic aneurysm, Combination therapy, Aspirin, Ticagrelor, Platelets

Posted Date: February 28th, 2022

DOI: https://doi.org/10.21203/rs.3.rs-1290930/v1

License: (c) (i) This work is licensed under a Creative Commons Attribution 4.0 International License.

Read Full License 
Combinational Therapy with Aspirin and Ticagrelor Alleviates Vascular Inflammation and Angiotensin II-driven Abdominal Aortic Aneurysm Formation in Mice

Xiaowei Liu ${ }^{1}$, Yingzheng Weng ${ }^{1}$, Jiangjie Lou ${ }^{1}$, Xiaofeng Chen ${ }^{2}$, Changqing Du ${ }^{1}$, Lijiang Tang 1

${ }^{1}$ Department of Cardiology, Zhejiang Hospital, Hangzhou, Zhejiang 310013, P. R. China.

${ }^{2}$ Department of Cardiology, Taizhou Hospital, Wenzhou Medical University, Taizhou, Zhejiang 317000, P. R. China.

Co-author: Xiaowei Liu, Yingzheng Weng and Jiangjie Lou have equally contributed to this study.

* Corresponding authors at: Lijiang Tang, Department of Cardiology, Zhejiang Hospital, 12 Lingying Road, Hangzhou, Zhejiang 310013, P. R. China; Xiaofeng Chen, Department of Cardiology, Taizhou Hospital, Wenzhou Medical University, 150 Ximen Street, Linhai 317000, P.R. China.

E-mail address: zjyytang@126.com (L-J. Tang); zjxiaofengchen@hotmail.com (X-F. Chen). 


\section{Abstract}

Background: Abdominal aortic aneurysm (AAA) is a severe form of blood vessel-related disease. Medial degeneration and inflammation are typical characteristic of AAA. Activated platelets release many pro-inflammatory cytokines and participate in the initial inflammatory response to various vascular diseases. Although there are some studies on the effects of APAs on AAA, it is yet unknown whether the new P2Y12 receptor inhibitor ticagrelor (T) can inhibit AAA. Herein, we explored the consequences of ticagrelor exposure on AAA progression and determined whether a combinational therapy, involving $\mathrm{T}$ and aspirin (A), exerts a stronger inhibitory effect in vivo.

Methods: AAA was established in apolipoprotein E-deficient $\left(\mathrm{ApoE}^{-/}\right)$mice via a 28-day administration of angiotensin II (Ang II). Next, the mice were arbitrarily separated into 5 groups: saline infusion alone (sham), Ang II infusion alone, Ang II infusion plus oral A (10 $\left.\mathrm{mg} \cdot \mathrm{kg}^{-1} \cdot \mathrm{d}^{-1}\right)$, Ang II infusion plus oral T (120 mg. $\left.\mathrm{kg}^{-1} \cdot \mathrm{d}^{-1}\right)$, Ang II infusion plus combinational therapy with A $\left(10 \mathrm{mg} \cdot \mathrm{kg}^{-1} \cdot \mathrm{d}^{-1}\right)$ and $\mathrm{T}\left(120 \mathrm{mg} \cdot \mathrm{kg}^{-1} \cdot \mathrm{d}^{-1}\right)$.

Results: The combined treatment markedly suppressed the Ang II-driven elevation of maximal aortic diameter, aneurysm formation $(26.7 \%$ decrease, $P<0.05)$, alterations in aortic expansion, elastic lamina destruction, platelet deposition, and inflammatory cytokine accumulation. In addition, it also diminished matrix metalloproteinase (MMP)-2 and MMP-9 production.

Conclusions: A combinational therapy of $\mathrm{A}$ and $\mathrm{T}$, but not individual drugs, inhibits Ang II-driven AAA generation in mice in vivo, and this process may be regulated by a suppressed inflammatory response.

Keywords: Abdominal aortic aneurysm, Combination therapy, Aspirin, Ticagrelor, Platelets 


\section{Introduction}

Abdominal aortic aneurysm (AAA) occurs in roughly 5-10\% males and $1 \%$ females, aged $>65$ years, and is considered the $13^{\text {th }}$ major causes of disease-related deaths in the United States [1,2]. According to the "Global Burden of Disease 2010" project, the global mortality rate of AAA went from 2.49 cases to 2.78 cases per 100,000 inhabitants between 1990 and 2010, and the mortality rate was higher in men [3,4]. AAA is the persistence of a local arterial expansion $(>50 \%)$ [5], followed by rupture. AAA pathology involves biochemical, cellular, proteolytic, and biomechanical factors that can result in regionalized inflammation of the arterial wall, which eventually leads to the destruction of extracellular matrix (ECM) proteins. Multiple reports have suggested that the aortic wall infiltration of inflammatory cells (ICs) like T cells, macrophages, neutrophils and dendritic cells is a definite pathogenic promoter of AAA [6,7].

Platelets are essential for hemostasis. However, its blood clotting mechanism can also promote thrombosis, inflammation, and endothelial dysfunction. Additionally, platelets are known to modulate inflammatory responses in vascular diseases $[8,9]$. Patients at risk of thrombosis are often managed with platelet inhibitors (PI) like hromboxane $\mathrm{A}_{2}$ and $\mathrm{P} 2 \mathrm{Y}_{12}$ receptor inhibitors. Based on a study by Dai et al., platelet activation strongly modulates AAA progression [10]. Importantly, plasma platelets and platelet-specific secretions are evident in AAA patients and are particularly released from the intraluminal thrombus in AAA [11,12]. Although there are some explorations into the effects of antiplatelet drugs (APA) on AAA $[13,14]$, nothing is known about the ability of the new P2Y12 receptor inhibitor ticagrelor $(\mathrm{T})$ in suppressing AAA. T is a novel P2Y12 receptor inhibitor, which was first introduced in the platelet inhibition and patient outcomes (PLATO) study [15]. Compared to traditional P2Y12 receptor inhibitor clopidogrel, $\mathrm{T}$ is known to produce a stronger antiplatelet activity, which can dramatically diminish cardiovascular death by $21 \%$ [15]. Subsequently, several reports demonstrated that $\mathrm{T}$ also has a strong anti-inflammatory property. Given these evidences, herein, we examined the consequences of $\mathrm{T}$ therapy on AAA progression. We also determined whether a combined treatment of $\mathrm{T}$ and A can produce an even stronger anti-AAA effect.

Our goal was to explore the dual antiplatelet and anti-inflammatory roles of $\mathrm{T}$ and combinational ( $\mathrm{T}$ and $\mathrm{A}$ ) therapies on AAA progression in a murine AAA model Upon AAA 
establishment in mice, platelet deposition was evident in the aortic wall. Among all examined treatments, the combined treatment of $\mathrm{T}$ and A produced the largest decrease in AAA progression, as evidenced by the marked reduction in the ICs recruitment, reactive inflammatory factors production, and matrix metalloproteinases (MMPs) activation within the aortic wall.

\section{Methods}

\section{Ethical Statement}

Our animal care and experimental protocols received approval from the Zhejiang Academy of Medical Sciences (Hangzhou, China). All experiments relating to animals followed the Guide for the Care and Use of Laboratory Animals published by the US National Institutes of Health (NIH Publication No. 85-23, revised 1996). Besides, our study is reported in accordance with ARRIVE guidelines.

\section{Animals}

$A p o E^{-/-}$mice on a C57BL/6 background were acquired from the Biomedicine of Nanjing Institute (Nanjing, China) and were bred in-house at the Medical Sciences of Zhejiang Academy (Hangzhou, China) and fed the standard mouse chow diet.

\section{Drugs}

70 mice, aged $8-10$ weeks, were arbitrarily assigned to 5 experimental groups as follows: Group 1 (G1): normal saline (sham, $n=10$ ); Group 2 (G2): Angiotensin II (Ang II, 1,000 ng/kg/min, Sigma, St. Louis, MO) with methylcellulose treatment (Ang II, $n=15$ ); Group 3 (G3): Ang II plus oral A (ASA, $10 \mathrm{mg} \cdot \mathrm{kg}^{-1} \cdot \mathrm{d}^{-1}$ ) (Ang II+ASA, $n=15$ ), and the oral A dose was according to the Liu's study [16]; Group 4 (G4): Ang II plus oral T (TIC, $120 \mathrm{mg} \cdot \mathrm{kg}^{-1} \cdot \mathrm{d}^{-1}$ ) (Ang II+TIC, $n=15$ ), and the oral T dose was according to the Ganbaatar 's study [17]; Group 5 (G5): Ang II with A and T combinational therapy (Ang II + Combi, $n=15$ ). Both A (Sigma Aldrich) and T (AstraZeneca, London, UK) were prepared as suspensions in $0.5 \%$ methylcellulose prior to a daily administration via gavage.

\section{Implantation of mini-osmotic pumps}


Following a 7-day gavage-infusion therapy of each drug, male mice received Alzet osmotic minipumps (Model 2004, Durect Corporation, Cupertino, CA, USA) implantation on the back of the neck. The implantation allowed for the continuous subcutaneous infusion of Ang II or saline vehicle, at a concentration of $1,000 \mathrm{ng} / \mathrm{kg} / \mathrm{min}$, as reported previously [18]. Body weight was measured weekly throughout the experiment.

\section{Blood pressure (BP) measurement}

Systolic BP (SBP), diastolic BP (DBP), and the heart rate (HR) were recorded via a noninvasive tail-cuff instrument (BP-98A, Softron, Tokyo, Japan), as reported previously [19]. BP was collected on the 1 week prior to the minipump implantation and on the last 7 days of the study.

\section{Western blotting}

Total protein from abdominal aortic tissues was isolated. MMP-2 and MMP-9 protein levels were examined via western blot. Protein concentration was quantified with BCA assay. $30 \mu \mathrm{g}$ of protein was separated on a $10 \%$ sodium dodecyl sulfate-polyacrylamide gel electrophoresis (SDS-PAGE) and transferred to polyvinylidene difluoride (PVDF) membranes, before blocking with $10 \%$ non-fat milk in Tris buffer solution with $0.1 \%$ Tween-20 (TBST) for 1 hour at room temperature (RT) with slight agitation, followed by overnight $(\mathrm{O} / \mathrm{N})$ incubation with anti-MMP-2 and anti-MMP-9 antibodies (Abcam) at $4^{\circ} \mathrm{C}$, three rinses for 10 minutes each, followed by a 1 hour incubation at RT with goat anti-rabbit IgG antibodies (Cell Signaling, Danvers, MA, USA), prepared at a dilution of 1:3000 in blocking solution, and lastly, three more rinses with TBST for 10 minutes, before protein visualization using an enhanced chemiluminescence (ECL) detection reagent (Beit Haemek LTD, kibbutz, Israel), ECL peroxidase substrate (MILLIPORE, Billerico, USA), and molecular imaging system (BIO RAD, CA, USA). GAPDH was employed as loading control. Lastly, protein quantification was done with Image J (Media Cybernetics, Houston, TX, USA).

\section{Histology and immunohistochemistry}

To evaluate vascular morphology, mice were treated as indicated above and then provided with 3.0\% isoflurane (anaesthetics). Next, the heart was punctured and perfused with PBS. Blood was 
collected for further analysis and $10 \%$ formalin was infused into the heart at a physiological rate using an infusion pump (Harvard apparatus, Holliston, MA, USA), followed by a 48-hour fixation of the entire aorta, from the ascending aorta to the iliac bifurcation, with $4 \%$ paraformaldehyde. The maximal abdominal aortic diameter (MAAD) represented the abdominal aortic diameter, and it measured by the Image-Pro Plus 5.0 analysis software (Media Cybernetics, Houston, TX, USA). Subsequently, the aorta was sliced into $3-\mu \mathrm{m}$ sections, before paraffin embedding and were either stained with Hematoxyln and eosin (H\&E) (aortic shape and arrangement evaluation), Masson's trichrome (collagen fibrosis evaluation), or van Gieson (elastin evaluation), following operational directions.

Some abdominal aortic sections also received $\mathrm{O} / \mathrm{N}$ exposure at $4^{\circ} \mathrm{C}$ to primary antibodies against CD41 (1:100), CD8 (1:200), CD40 (1:200), CD68 (1:200), MMP2 (1:1000), and MMP9 (1:1000; all Abcam, Cambridge, MA); as well as monocyte chemoattractant protein 1 (MCP-1; 1:400), interleukin 1 (IL-1; 1:400), and tumor necrosis factor- $\alpha$ (TNF- $\alpha$; 1:400; all Santa Cruz Biotechnology, Santa Cruz, CA). The negative control (NC) samples either did not receive any primary antibody exposure or only received goat non-immune $\operatorname{IgG}$, rabbit non-immune $\operatorname{IgG}$ or secondary antibody. In all cases, the $\mathrm{NC}$ was not significantly stained. Immunostaining quantification was done while blinded to the treatment conditions.

\section{MAAD measurement}

The murine aorta was placed under a stereo microscope (Antaixing Electronics Company, Shenzhen, China) after sufficient fixation in $4 \%$ paraformaldehyde. Next, the muscle and adipose tissue around the artery were cleaned, and the kidney artery was reserved for AAA positioning, and lastly, the images were captures against a dark background. A scale was included with each photograph. . Finally, the MAAD was measured as mentioned before.

AAA was described as $\geq 50 \%$ expansion of MAAD. Necropsy was conducted immediately after any animal died before sacrifice. Given the presence of tissue degradation, the animals that died before sacrifice were eliminated from the histological analysis, but included in the mortality analysis.

\section{Statistical analysis}


Data are expressed as mean \pm standard deviation (SD). Multi-group analysis was done with 1-way Analysis of Variance (ANOVA), followed by a Tukey-Kramer post hoc test. Variance homogeneity was verified with the $F$ test, whereas the Tamhane's T2 test was employed for data with unequal variance. Two-group analysis was done with Student's $t$ test. $P<0.05$ was set as the significance threshold. SAS version 9.3 (SAS Institute, Cary, North Carolina) was employed for the analysis of all data.

\section{Results}

APDs prolongs bleeding duration but does not affect SBP, lipid profile, or platelet count in mice

To examine the relationship between platelet deposition and AAA development, we suppressed platelet activity in a murine Ang II-administered AAA model, using APD, namely, a regimen of A, $\mathrm{T}$, or a combination of A and T. Our data demonstrated that APA markedly enhanced bleeding duration in the AAA mice, as compared to AAA mice receiving no APD (Ang II only, 110.2 \pm 25.8

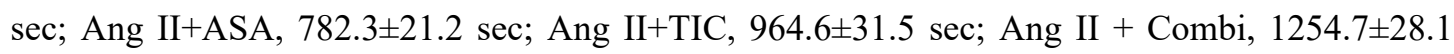
sec). (Table 1).

A 28-day Ang II infusion markedly elevated murine SBP from $92.6 \pm 4.7$ to $143.6 \pm 3.7 \mathrm{~mm} \mathrm{Hg}$ (Table 1). However, mice $\mathrm{BP}$ remained unaffected. All $\mathrm{ApoE}^{-/}$mice exhibited severe hyperlipidemia. However, neither Ang II administration nor APD altered the cholesterol or triglycerides levels or platelet count in mice at the end of the study (Table 1).

\section{Combinational therapy with A and T blocks Ang II-induced AAA formation}

Sham mice had no AAA formation (Table 1 and Fig. 1A). The combined A and T treatment strongly blocked Ang II-driven rise in AAA incidence (Fig. 1B) and MAAD (sham, 1.01 \pm 0.08 mm; Ang II, $2.34 \pm 0.31 \mathrm{~mm}$; A, $2.24 \pm 0.32 \mathrm{~mm}$; T, $2.28 \pm 0.29 \mathrm{~mm}$; combination, $1.32 \pm 0.10 \mathrm{~mm}$; $P<0.01$, Ang II vs. Ang II + Combi) and (Fig. 1C). Based on the pathological staining (Fig. 2A D), the elastic lamellae were badly damaged in the Ang II mice, relative to the sham mice. This affect was obviously blocked in the Ang II + Combi mice, but not in mice receiving individual drug therapies. There were no deaths among the sham mice. Additionally, no marked differences were observed in aortic rupture-related mortality, as evidenced by necroscopy [Ang II, 41.7\% 
(5/12); Ang II+ASA, 36.4\% (4/11); Ang II+TIC, 45.5\% (5/11); Ang II + Combi, 37.5\% (3/8)] (Table 1 and Fig. 1D).

\section{Combination therapy with $A$ and $T$ decreases platelets deposition and vascular inflammation in mice}

To delineate the mechanisms involving the platelet-mediated modulation of AAA progression, we examined aortic platelet deposition, in presence or absence of AAA. Based on our analysis, massive platelet deposition occurred in the aorta of AAA, relative to non-AAA mice as evidenced by immunostaining of the platelet-specific marker CD41 (Fig. 3A\&B), and platelet deposition reduced dramatically with $\mathrm{A}$ and $\mathrm{T}$ combinational therapy. Vascular inflammation is critical to AAA development, and inflammatory cell infiltration is indispensible to the inflammatory process. Hence, to explore the role of antiplatelets in regulating AAA-mediated vascular inflammation, we evaluated inflammatory cell infiltration in serial tissue sections collected from the same distance from the point of MAAD. As depicted in Figures 4, the Ang II mice exhibited CD40-positive B lymphocytes, CD8-positive T lymphocytes, and CD68-positive macrophages infiltration into the adventitia and aortic regions. In contrast, the combined therapy markedly inhibited macrophage, T-lymphocytes and B-lymphocytes accumulation. In fact, the inflammatory cell infiltration in combined treated mice was relatively similar to the Sham mice $(P<0.05)$, whereas A or $\mathrm{T}$ monotherapy failed to reduce lymphocytes and macrophage infiltration, relative to the Ang II mice.

To delineate the mechanism whereby platelet suppression modulates inflammatory response, we compared the release of inflammatory factors in the aortic wall to macrophage recruitment and AAA development. The combinational therapy strongly diminished MCP-1, IL- $1 \beta$, and TNF- $\alpha$ accumulation in the aortas of Ang II-treated mice (Fig. 5). However, this was not the case in terms of $\mathrm{A}$ or $\mathrm{T}$ monotherapy. Hence, a combined treatment, involving $\mathrm{A}$ and $\mathrm{T}$, produces the most satisfactory suppression of vascular inflammatory response in AAA.

\section{Combinational A and T therapy prevents aortic Ang II-induced MMPs synthesis}

MMPs modulate AAA formation and aortic rupture. Given that the combined treatment successfully reduced macrophage infiltration, we speculated that it would also downregulate MMP 
expressions. To test this, we assessed the levels of murine aortic MMP-2 and MMP-9 levels, in presence or absence of antiplatelet therapy (Fig. 6). We demonstrated that, as expected, both MMP-2 and MMP-9 secretions were markedly diminished in the aorta of Ang II-infused ApoE $E^{-/}$ mice that underwent combined treatment $(P<0.05)$. In contrast, $\mathrm{A}$ or $\mathrm{T}$ monotherapy did not alter MMP-2 or MMP-9 levels, relative to the Ang II mice.

\section{Discussion}

Inflammation is a major cause of AAA development. In recent years, more and more evidence showed that platelets are an important component of the inflammatory immune system. Herein, we demonstrated the presence of massive populations of platelet and ICs in the arteries of a murine AAA model. Furthermore, we revealed that combined treatment with the APAs A and T can remarkably decrease macrophage infiltration, MMPs expression, and induce a $26.7 \%$ reduction $(P<0.05)$ in AAA development. However, of note, no discernible differences were observed in aneurysm rupture prevalence in mice treated or untreated with APD.

A distinguishing characteristic of human AAA is the presence of the layered wall thickness platelet thrombus that develops along the lumen surface area [20,21]. Importantly, clinical studies demonstrated that the amount of blood clots or blood replacement requirements that occur due to parietal thrombus may be a sign of AAA expansion and rupture [22]. Additionally, in a rat AAA model, suppressing platelet activation via AZD6140 markedly reduced aneurysm formation [10]. These findings indicate that platelets are closely related to AAA. Platelets and platelet-based agents usually remain beyond hemostatic levels in vascular diseases like atherosclerosis and coronary artery disease (CAD) [23]. Platelets are an essential source of pro-inflammatory mediators and cytokines, which then recruit ICs like T cells and macrophages [24,25]. Prior research revealed that aortic vascular inflammation is a major cause of AAA progression. Moreover, the accumulation of ICs often enhances development of AAA lesions [26,27]. Similar to these evidences, we also observed a positive correlation between platelet deposition and inflammatory cell infiltration in our mice experiments.

A is recommended for AAA patients from diagnosis to perioperative period. However, clinical investigations on the consequences of platelet suppressor on non-hereditary AAA rupture and expansion are very limited. It is speculated that A works by reducing aneurysm expansion in 
part by alleviating aortic thrombosis and inflammation while stabilizing the aortic wall. Lindholt found that a low dose of A could significantly slow the rate of AAA expansion in AAA patients with diameter between 40-49mm [28]. However, a large meta-analysis revealed that APD may, in fact, produce no remarkable effect on AAA growth, relative to untreated aneurysms, after adjusting for confounding factors $[29,30]$. Similarly, we also demonstrated that individual drug treatment alone does not affect AAA growth in an established murine AAA model.

$\mathrm{T}$ is a new P2Y12 receptor inhibitor that is reported to have a more potent antiplatelet property than clopidogrel [15]. Prior reports have suggested that $T$ not only inhibits the systemic inflammatory response, but also has a therapeutic effect on the local inflammatory response of atherosclerotic plaque [31,32]. Interestingly, both pathological alterations and risk factors associated with AAA are comparable to that of atherosclerosis. Thus, we investigated whether T therapy alone, or in combination with A, can prevent AAA progression in a murine Ang IIinfused AAA model. This is a novel study exploring the effect of $\mathrm{T}$ on a murine AAA model. Unfortunately, the results were not exactly what we expected, $\mathrm{T}$ monotherapy did not effectively prevent the progression of AAA. However, our conclusion is in accordance with a recent randomized controlled trial, involving multiple centers [33]. In the aforementioned trial, the authors examined whether T could inhibit growth of small AAA with a maximum aortic diameter 35-49 $\mathrm{mm}$. They reported was no change in AAA volume or diameter, between the T and placebo groups, at the 12 months follow-up [33].

Emerging evidences have pointed to a possible synergistic effect when combining A with $\mathrm{T}$ in the treatment of vascular inflammation [34]. However, the consequences of such combinatory therapy in AAA regulation have never been explored. Based on our analysis, combined A and T therapy, but not individual APD, can strongly block Ang II-driven AAA development in mice. It is possible that the mechanism of action of the combined drug therapy involves suppression of the inflammatory response. First, we demonstrated that platelet deposition is markedly elevated in AAA lesions, as was shown in other publications [13], and this rise in platelet deposition was vastly abrogated via combined treatment. In prior studies, it was shown that the communication between platelets and ICs largely contributes to the occurrence and development of AAA [13]. In accordance with these publications, we also observed a strong correlation between platelet accumulation and inflammatory cell infiltration in mice (Fig. $3 \&$ Fig. 4). In addition, with 
enhanced macrophage infiltration, there also was a dramatic rise in the aortic MCP-1 levels of Ang II-treated mice. However, combined treatment also abrogated these alterations. Second, matrix destruction is another crucial step to AAA development. Inflammatory cell infiltration produces localized inflammation via the release of cytokines, chemokines, oxygen free radicals, and MMPs [7]. Thus, MMP release can also contribute to the occurrence, development, and complications of AAA [35]. Our analysis revealed that both aortic MMP-2 and MMP-9 synthesis were diminished after combined treatment (Figure 5). Lastly, despite combination therapy significantly reducing inflammation-related AAA lesions, the underlying mechanism behind its synergistic inhibition remains to be explored.

This study had certain limitations. First, AAA was established in the superior renal artery in our Ang II-stimulated murine AAA model. However, typically, AAA occurrence is primarily limited to the human infrarenal abdominal aorta. Therefore, the current findings require further validation in other AAA models. Second, mice and human may have vastly different pharmacokinetics for A and T. Thus, our conclusions should be further validated in clinical trials. Lastly, the underlying mechanism of action of the proposed combinational therapy is still unknown. Therefore, future research should focus on the specific mechanism involving this phenomenon.

\section{Conclusions}

In conclusion, our work was the first to report the synergistic beneficial effect of $\mathrm{A}$ and $\mathrm{T}$ combinational therapy in suppressing Ang II-driven AAA development in mice and suppression of inflammatory response may be involved in this process.

\section{Acknowledgements}

Not applicable.

\section{Authors' contributions}

X-F.C. and L-J.T. revised the manuscript, conceived and designed the experiments; X-W.L., J-J.L. and Y-Z.W. wrote the paper, analyzed the data and performed the experiments; C-Q.D. contributed reagents, materials, and analysis tools. 


\section{Fundings}

The Major Research and Development Projects for the Zhejiang Science and Technology Agency (Grant No. 2017C03034), the National Natural Science Foundation of China (Grant No. 81450034 \& 81770475), the Scientific and Technological Projects for Medicine and Health of Zhejiang Province (Grant No. 2022481190 \& 2021KY426), the Natural Science Foundation of Zhejiang Province (Grant No. LY21H020002), the Major medical and health science and technology plan of Zhejiang Province (Grant No. WKJ-ZJ-1913).

\section{Availability of data and materials}

The datasets generated and analyzed during the current study are not publicly available due personal reasons but are available from the corresponding author on reasonable request.

\section{Declarations}

\section{Ethics approval and consent to participate}

Our animal care and experimental protocols received approval from the Zhejiang Academy of Medical Sciences (Hangzhou, China). All experiments relating to animals followed the Guide for the Care and Use of Laboratory Animals published by the US National Institutes of Health (NIH Publication No. 85-23, revised 1996).

\section{Consent for publication}

Not applicable.

\section{Competing interests}

The authors declare no conflict of interest. 


\section{Figure Legends}

Fig. 1. Combination therapy with aspirin (ASA) and ticagrelor (TIC) suppresses angiotensin II (Ang II)-induced abdominal aortic aneurysm (AAA) formation in $\mathrm{ApoE}^{-/-}$mice. A: Representative photographs showing macroscopic features of aortas from mice treated with saline (left), Ang II, Ang II + aspirin (Ang II+ASA), Ang II + ticagrelor (Ang II+TIC), and Ang II + aspirin+ ticagrelor (Ang II +Combi). B: The incidence of Ang II-induced AAA in different groups. C: Maximal abdominal aortic diameter in $A p o E^{-/-}$mice after Ang II infusion for 4 weeks. D: Percentage rupture of AAA during the experiment.

AAA formation: AAA is defined as $\geq 50 \%$ enlargement of the maximal abdominal aorta diameter. AAA rupture: Necropsy was performed as soon as the animals expired before sacrifice, and the abdominal aorta was verified by a microscope if a hematoma was found in the abdominal cavity of the mice; it is defined as a ruptured aneurysm when an aneurysm was found with a breach.

Data are presented as mean $\pm \mathrm{SD} ; n=10-15$ per group.

Fig. 2. Combination therapy ameliorates pathological features at the angiotensin II (Ang II)-induced abdominal aortic aneurysm (AAA) in $\mathrm{ApoE}^{-/-}$mice. A\&B: Representative photographs of Hematoxylin-Eosin (H\&E) staining. C: Micrographs of aortic sections stained with Masson trichrome stain: vascular smooth muscle cells (VSMCs) are red and collagen is green. D: Elastin van Gieson staining of aortic cross-sections of 5 groups. Abbreviations: ASA, aspirin; TIC, ticagrelor; Combi, combination.

Data are presented as mean $\pm \mathrm{SD} ; n=5-6$ per group.

Fig. 3. Combination therapy suppresses platelets deposition at the angiotensin II (Ang II)-induced abdominal aortic aneurysm (AAA) in $\mathrm{ApoE}^{-/-}$mice. A: Immunostaining for CD41(a specific marker for platelet). B: Quantitative analysis of CD41 positive cells. Abbreviations: ASA, aspirin; TIC, ticagrelor; Combi, combination.

Data are presented as mean $\pm \mathrm{SD} ; n=6-8$ per group.

Fig. 4. Combination therapy suppresses inflammatory cells infiltration at the angiotensin II (Ang II)-induced abdominal aortic aneurysm (AAA) in $\mathrm{ApoE}^{-/-}$mice. A: Immunostaining for CD40 (B 
lymphocytes maker), CD8 (T lymphocyte maker) and CD68 (a specific marker for mature macrophages). B-D: Quantitative analysis of CD40, CD8 and CD68 positive cells. Abbreviations: ASA, aspirin; TIC, ticagrelor; Combi, combination.

Data are presented as mean $\pm \mathrm{SD} ; n=6-8$ per group.

Fig. 5. Combination therapy suppresses inflammatory factors expression at the angiotensin II (Ang II)-induced abdominal aortic aneurysm (AAA) in $\mathrm{ApoE}^{-/-}$mice. A: Immunostaining for interleukin-1 $\beta$ (IL-1 $\beta)$, tumor necrosis factor- $\alpha$ (TNF- $\alpha$ ), and monocyte chemoattractant protein-1 (MCP-1). B-D: Quantitative analysis of IL-1 $\beta$, TNF- $\alpha$ and MCP-1, respectively. Abbreviations: ASA, aspirin; TIC, ticagrelor; Combi, combination.

Data are presented as mean $\pm \mathrm{SD} ; n=5-6$ per group.

Fig. 6. Combination therapy suppresses matrix metalloproteinases (MMPs) expression at the angiotensin II (Ang II)-induced abdominal aortic aneurysm (AAA) in $\mathrm{ApoE}^{-/-}$mice. A: Immunostaining for MMP-2 and MMP-9. B\&C: Quantitative analysis of MMP-2 and MMP-9, respectively. D: Representative Western blot band of MMP-2 and MMP-9. E\&F: Western blot analysis of MMP-2 and MMP-9. Abbreviations: ASA, aspirin; TIC, ticagrelor; Combi, combination.

Data are presented as mean $\pm \mathrm{SD} ; n=5$ per group. 


\section{References:}

[1] Powell JT. Abdominal Aortic Aneurysm Repair in England and the United States. N Engl J Med. 2017;376: 998 .

[2] Alcorn HG, Wolfson SJ, Sutton-Tyrrell K, Kuller LH, O'Leary D. Risk factors for abdominal aortic aneurysms in older adults enrolled in The Cardiovascular Health Study. Arterioscler Thromb Vasc Biol. 1996;16: 963-70.

[3] Murray CJ, Richards MA, Newton JN, Fenton KA, Anderson HR, Atkinson C, et al. UK health performance: findings of the Global Burden of Disease Study 2010. LANCET. 2013;381: 997-1020.

[4] Sampson UK, Norman PE, Fowkes FG, Aboyans V, Yanna S, Harrell FJ, et al. Global and regional burden of aortic dissection and aneurysms: mortality trends in 21 world regions, 1990 to 2010. Glob Heart. 2014;9: 171-80.

[5] Erbel R, Aboyans V, Boileau C, Bossone E, Bartolomeo RD, Eggebrecht H, et al. 2014 ESC Guidelines on the diagnosis and treatment of aortic diseases: Document covering acute and chronic aortic diseases of the thoracic and abdominal aorta of the adult. The Task Force for the Diagnosis and Treatment of Aortic Diseases of the European Society of Cardiology (ESC). EUR HEART J. 2014;35: 2873-926.

[6] Ying AJ, Affan ET. Abdominal Aortic Aneurysm Screening: A Systematic Review and Meta-analysis of Efficacy and Cost. ANN VASC SURG. 2019;54: 298-303.

[7] Jiang H, Sasaki T, Jin E, Kuzuya M, Cheng XW. Inflammatory Cells and Proteases in Abdominal Aortic Aneurysm and its Complications. CURR DRUG TARGETS. 2018;19: 1289-96.

[8] Koupenova M, Clancy L, Corkrey HA, Freedman JE. Circulating Platelets as Mediators of Immunity, Inflammation, and Thrombosis. CIRC RES. 2018;122: 337-51.

[9] Vogel S, Thein SL. Platelets at the crossroads of thrombosis, inflammation and haemolysis. Br J Haematol. 2018;180: 761-7.

[10] Dai J, Louedec L, Philippe M, Michel JB, Houard X. Effect of blocking platelet activation 
with AZD6140 on development of abdominal aortic aneurysm in a rat aneurysmal model. J VASC SURG. 2009;49: 719-27.

[11] Kaur R, Singh J, Kapoor R, Kaur M. Association of SELP Polymorphisms with Soluble P-Selectin Levels and Vascular Risk in Patients with Type 2 Diabetes Mellitus: A Case-Control Study. BIOCHEM GENET. 2019;57: 73-97.

[12] Lareyre F, Carboni J, Chikande J, Massiot N, Voury-Pons A, Umbdenstock E, et al. Association of Platelet to Lymphocyte Ratio and Risk of 30-Day Postoperative Complications in Patients Undergoing Abdominal Aortic Surgical Repair. Vasc Endovascular Surg. 2019;53: 5-11.

[13] Liu O, Jia L, Liu X, Wang Y, Wang X, Qin Y, et al. Clopidogrel, a platelet P2Y12 receptor inhibitor, reduces vascular inflammation and angiotensin II induced-abdominal aortic aneurysm progression. PLOS ONE. 2012;7: e51707.

[14] Owens AR, Edwards TL, Antoniak S, Geddings JE, Jahangir E, Wei WQ, et al. Platelet Inhibitors Reduce Rupture in a Mouse Model of Established Abdominal Aortic Aneurysm. Arterioscler Thromb Vasc Biol. 2015;35: 2032-41.

[15] Wallentin L, Becker RC, Budaj A, Cannon CP, Emanuelsson H, Held C, et al. Ticagrelor versus clopidogrel in patients with acute coronary syndromes. N Engl J Med. 2009;361 : 1045-57.

[16] Liu PP, Liu HH, Sun SH, Shi XX, Yang WC, Su GH, et al. Aspirin alleviates cardiac fibrosis in mice by inhibiting autophagy. ACTA PHARMACOL SIN. 2017;38: 488-97.

[17] Ganbaatar B, Fukuda D, Salim HM, Nishimoto S, Tanaka K, Higashikuni Y, et al. Ticagrelor, a P2Y12 antagonist, attenuates vascular dysfunction and inhibits atherogenesis in apolipoprotein-E-deficient mice. ATHEROSCLEROSIS. 2018;275: 124-32.

[18] Bruemmer D, Collins AR, Noh G, Wang W, Territo M, Arias-Magallona S, et al. Angiotensin II-accelerated atherosclerosis and aneurysm formation is attenuated in osteopontin-deficient mice. J CLIN INVEST. 2003;112: 1318-31.

[19] Kanda T, Hayashi K, Wakino S, Homma K, Yoshioka K, Hasegawa K, et al. Role of Rho-kinase and p27 in angiotensin II-induced vascular injury. HYPERTENSION. 2005;45: 724-9.

[20] Fernandez-Garcia CE, Burillo E, Lindholt JS, Martinez-Lopez D, Pilely K, Mazzeo C, et al. 
Association of ficolin-3 with abdominal aortic aneurysm presence and progression. $\mathrm{J}$ THROMB HAEMOST. 2017;15: 575-85.

[21] Davies RS, Abdelhamid M, Vohra RK, Bradbury AW, Adam DJ. The relationship between aortic aneurysm sac thrombus volume on coagulation, fibrinolysis and platelet activity. THROMB RES. 2012;130: 463-6.

[22] Touat Z, Ollivier V, Dai J, Huisse MG, Bezeaud A, Sebbag U, et al. Renewal of mural thrombus releases plasma markers and is involved in aortic abdominal aneurysm evolution. AM J PATHOL. 2006;168: 1022-30.

[23] Lievens D, von Hundelshausen P. Platelets in atherosclerosis. Thromb Haemost. 2011;106: 827-38.

[24] Duchene J, von Hundelshausen P. Platelet-derived chemokines in atherosclerosis. HAMOSTASEOLOGIE. 2015;35: 137-41.

[25] Lim GB. Pro-inflammatory atherogenic role of platelets. NAT REV CARDIOL. 2020;17: 6-7.

[26] Kuivaniemi H, Ryer EJ, Elmore JR, Tromp G. Understanding the pathogenesis of abdominal aortic aneurysms. Expert Rev Cardiovasc Ther. 2015;13: 975-87.

[27] Ijaz T, Sun H, Pinchuk IV, Milewicz DM, Tilton RG, Brasier AR. Deletion of NF-kappaB/RelA in Angiotensin II-Sensitive Mesenchymal Cells Blocks Aortic Vascular Inflammation and Abdominal Aortic Aneurysm Formation. Arterioscler Thromb Vasc Biol. 2017;37: 1881-90.

[28] Lindholt JS, Sorensen HT, Michel JB, Thomsen HF, Henneberg EW. Low-dose aspirin may prevent growth and later surgical repair of medium-sized abdominal aortic aneurysms. Vasc Endovascular Surg. 2008;42: 329-34.

[29] Sidloff DA, Stather PW, Choke E, Bown MJ, Sayers RD. A systematic review and meta-analysis of the association between markers of hemostasis and abdominal aortic aneurysm presence and size. J VASC SURG. 2014;59: 528-35.

[30] Sweeting MJ, Thompson SG, Brown LC, Powell JT. Meta-analysis of individual patient data to examine factors affecting growth and rupture of small abdominal aortic aneurysms. $\mathrm{Br}$ J Surg. 2012;99: 655-65.

[31] Thomas MR, Outteridge SN, Ajjan RA, Phoenix F, Sangha GK, Faulkner RE, et al. Platelet 
P2Y12 Inhibitors Reduce Systemic Inflammation and Its Prothrombotic Effects in an Experimental Human Model. Arterioscler Thromb Vasc Biol. 2015;35: 2562-70.

[32] Oh M, Lee CW, Lee HS, Chang M, Ahn JM, Park DW, et al. Similar Impact of Clopidogrel or Ticagrelor on Carotid Atherosclerotic Plaque Inflammation. CLIN CARDIOL. 2016;39: 646-52.

[33] Wanhainen A, Mani K, Kullberg J, Svensjo S, Bersztel A, Karlsson L, et al. The effect of ticagrelor on growth of small abdominal aortic aneurysms-a randomized controlled trial. CARDIOVASC RES. 2020;116: 450-6.

[34] Kunadian V, Chan D, Ali H, Wilkinson N, Howe N, McColl E, et al. Antiplatelet therapy in the primary prevention of cardiovascular disease in patients with chronic obstructive pulmonary disease: protocol of a randomised controlled proof-of-concept trial (APPLE COPD-ICON 2). BMJ OPEN. 2018;8: e20713.

[35] Maguire EM, Pearce S, Xiao R, Oo AY, Xiao Q. Matrix Metalloproteinase in Abdominal Aortic Aneurysm and Aortic Dissection. Pharmaceuticals (Basel). 2019;12: . 


\section{Figures}

A

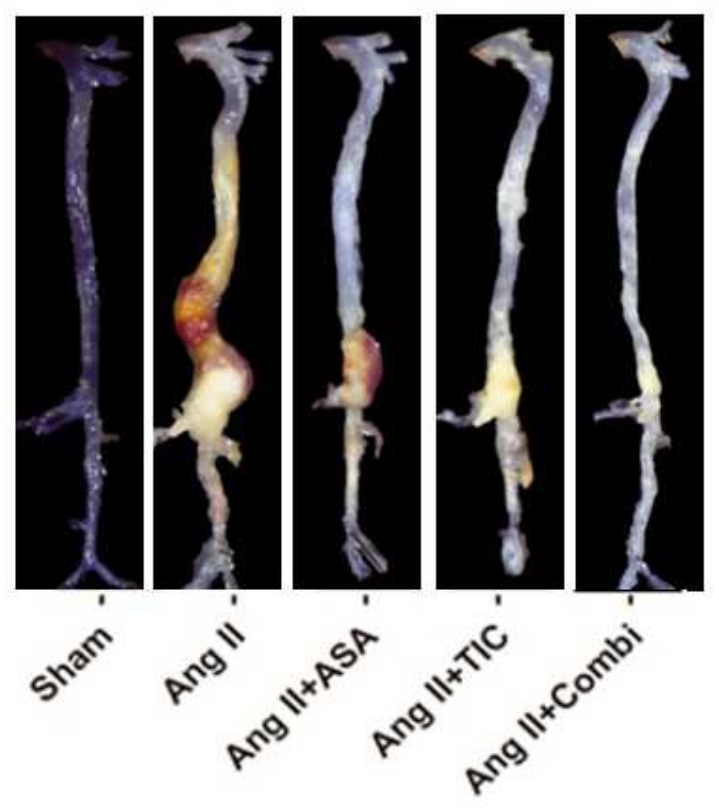

B

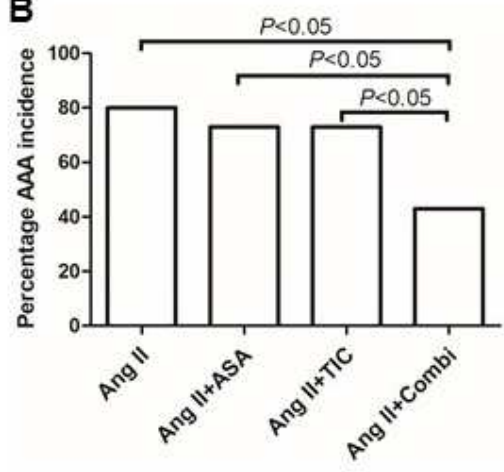

C

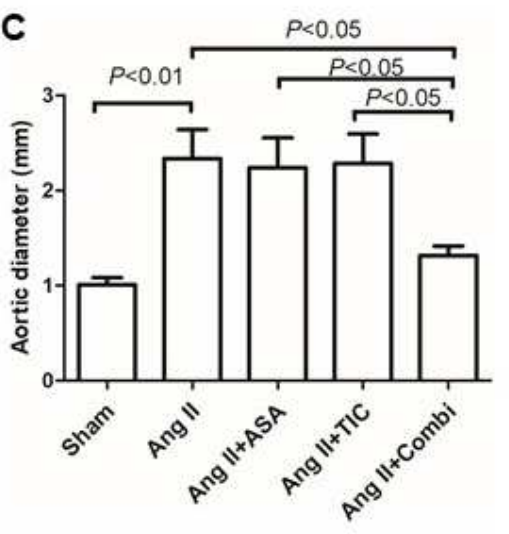

D

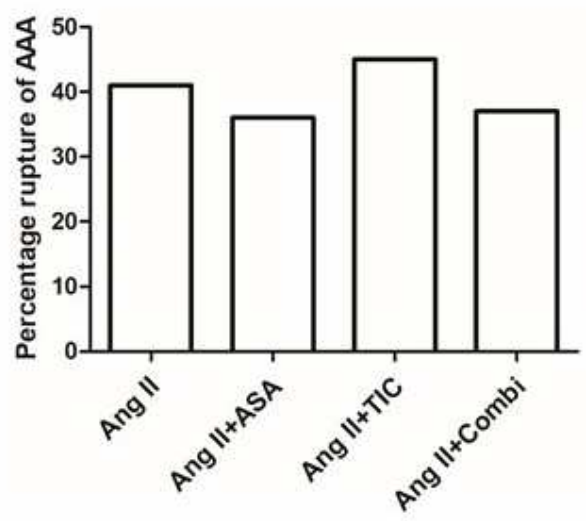

Figure 1

Combination therapy with aspirin (ASA) and ticagrelor (TIC) suppresses angiotensin II (Ang II)-induced abdominal aortic aneurysm (AAA) formation in ApoE-/- mice. A: Representative photographs showing macroscopic features of aortas from mice treated with saline (left), Ang II, Ang II + aspirin (Ang II+ASA) , Ang II + ticagrelor (Ang II+TIC), and Ang II + aspirin+ ticagrelor (Ang II +Combi). B: The incidence of Ang IIinduced AAA in different groups. C:

Maximal abdominal aortic diameter in ApoE-/- mice after Ang II infusion for 4 weeks. D: Percentage rupture of AAA during the experiment. AAA formation: AAA is defined as $\geq 50 \%$ enlargement of the maximal abdominal aorta diameter. AAA rupture: Necropsy was performed as soon as the animals expired before sacrifice, and the abdominal aorta was verified by a microscope if a hematoma was found in the abdominal cavity of the mice; it is defined as a ruptured aneurysm when an aneurysm was found with a breach.

Data are presented as mean \pm SD; $n=10-15$ per group. 


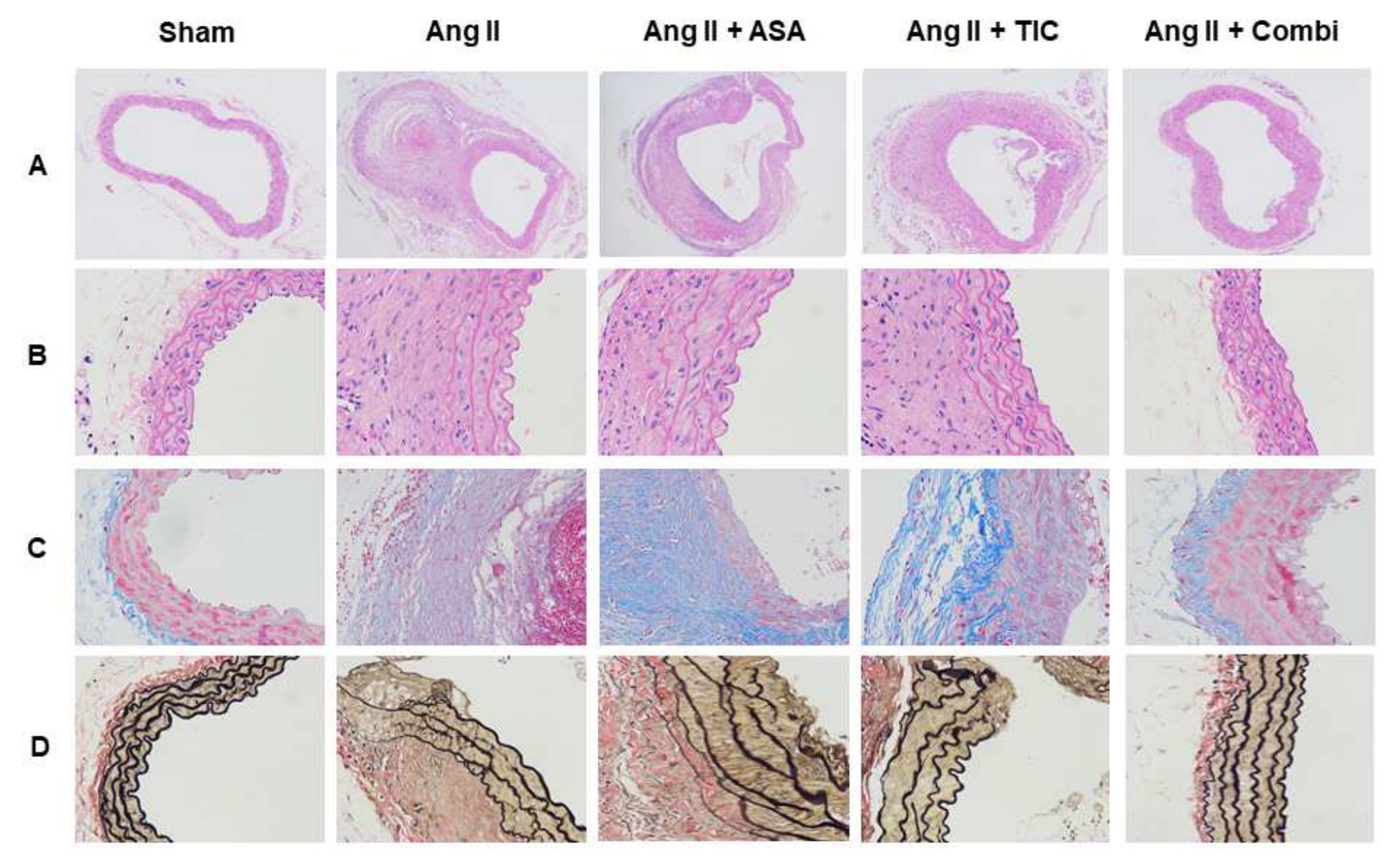

Figure 2

Combination therapy ameliorates pathological features at the angiotensin II (Ang II)-induced abdominal aortic aneurysm (AAA) in ApoE-/- mice. A\&B: Representative photographs of Hematoxylin-Eosin (H\&E) staining. C: Micrographs of aortic sections stained with Masson trichrome stain: vascular smooth muscle cells (VSMCs) are red and collagen is green. D: Elastin van Gieson staining of aortic cross-sections of 5 groups. Abbreviations: ASA, aspirin; TIC, ticagrelor; Combi, combination.

Data are presented as mean $\pm S D ; n=5-6$ per group.
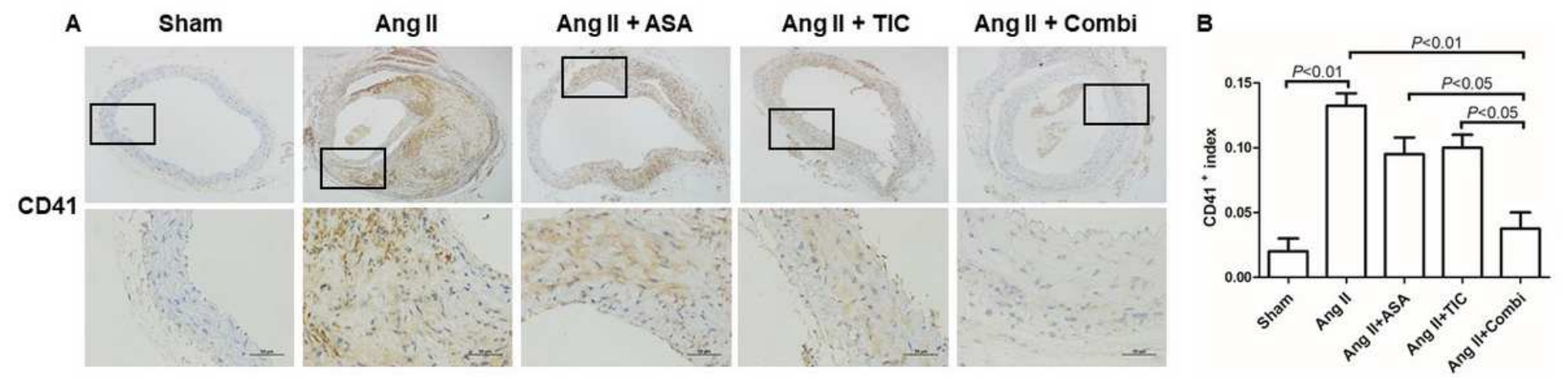

Figure 3 
Combination therapy suppresses platelets deposition at the angiotensin II (Ang II)-induced abdominal aortic aneurysm (AAA) in ApoE-/- mice. A: Immunostaining for CD41(a specific marker for platelet). B: Quantitative analysis of CD41 positive cells. Abbreviations: ASA, aspirin; TIC, ticagrelor; Combi, combination.

Data are presented as mean $\pm S D ; n=6-8$ per group.

A

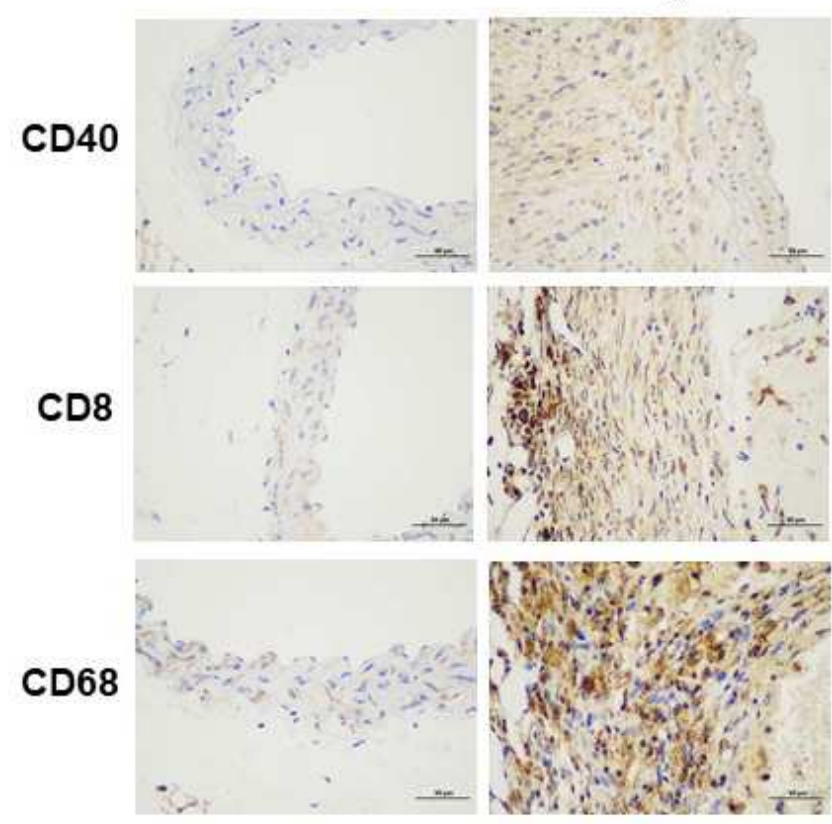

B

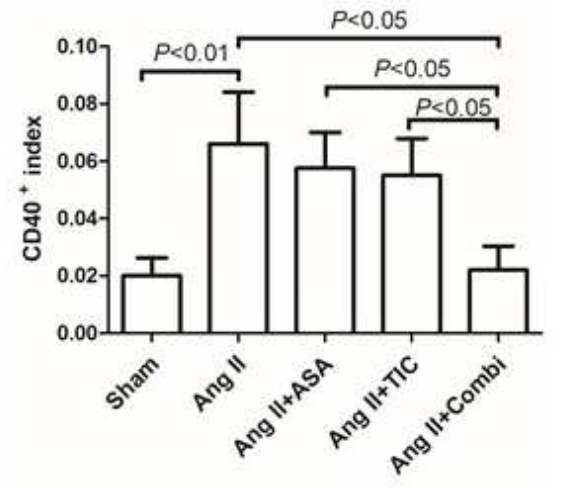

Ang II + ASA
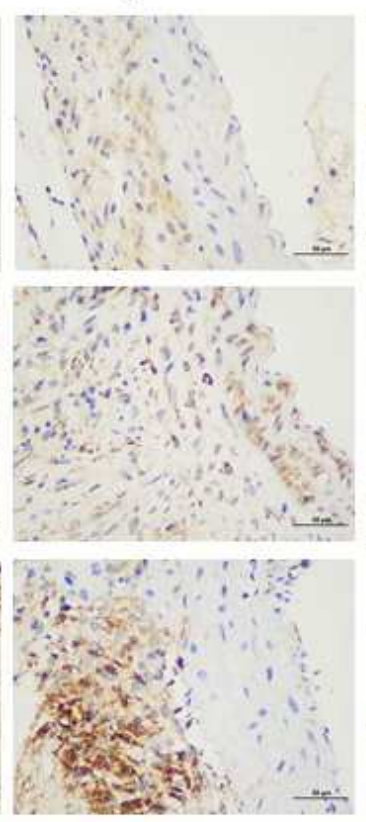

C

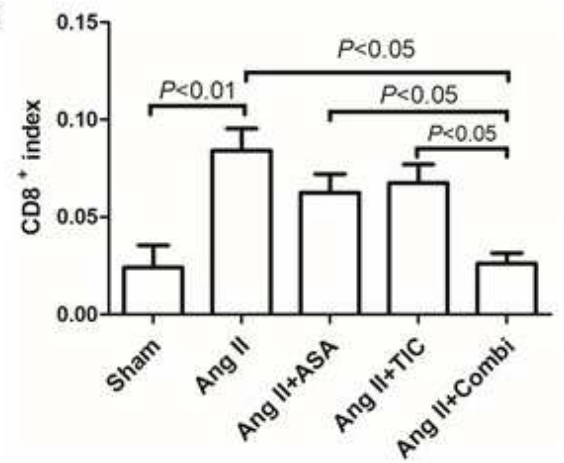

Ang II + TIC
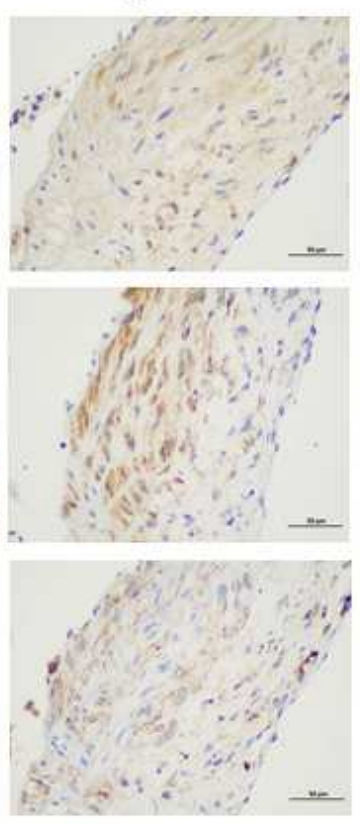

Ang II + Combi

D

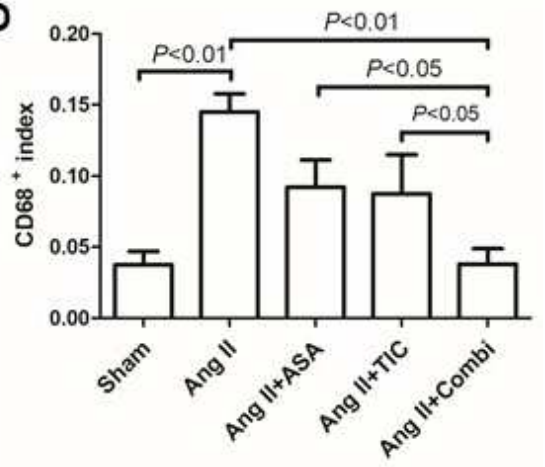

\section{Figure 4}

Combination therapy suppresses inflammatory cells infiltration at the angiotensin II (Ang II)-induced abdominal aortic aneurysm (AAA) in ApoE-/- mice. A: Immunostaining for CD40 (B lymphocytes maker), CD8 (T lymphocyte maker) and CD68 (a specific marker for mature macrophages). B-D: Quantitative analysis of CD40, CD8 and CD68 positive cells. Abbreviations:

ASA, aspirin; TIC, ticagrelor; Combi, combination.

Data are presented as mean $\pm S D ; n=6-8$ per group. 


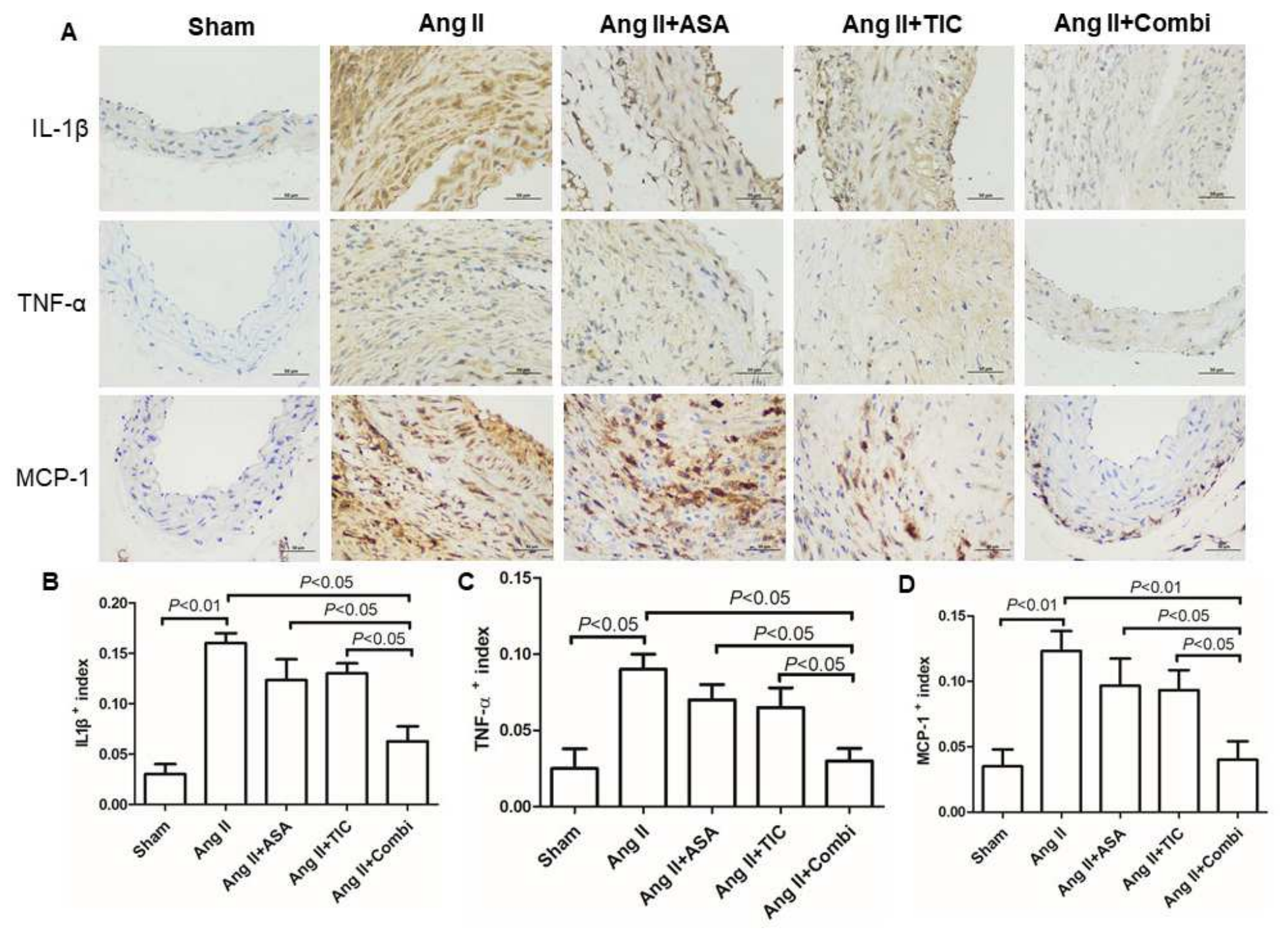

Figure 5

Combination therapy suppresses inflammatory factors expression at the angiotensin II (Ang II)-induced abdominal aortic aneurysm (AAA) in ApoE-/- mice. A: Immunostaining for interleukin-1 $\beta$ (IL-1 $\beta$ ), tumor necrosis factor-a (TNF-a), and monocyte chemoattractant protein-1 (MCP-1). B-D: Quantitative analysis of IL-1 $\beta$, TNF- $\alpha$ and MCP-1, respectively. Abbreviations: ASA, aspirin; TIC, ticagrelor; Combi, combination.

Data are presented as mean $\pm S D ; n=5-6$ per group. 


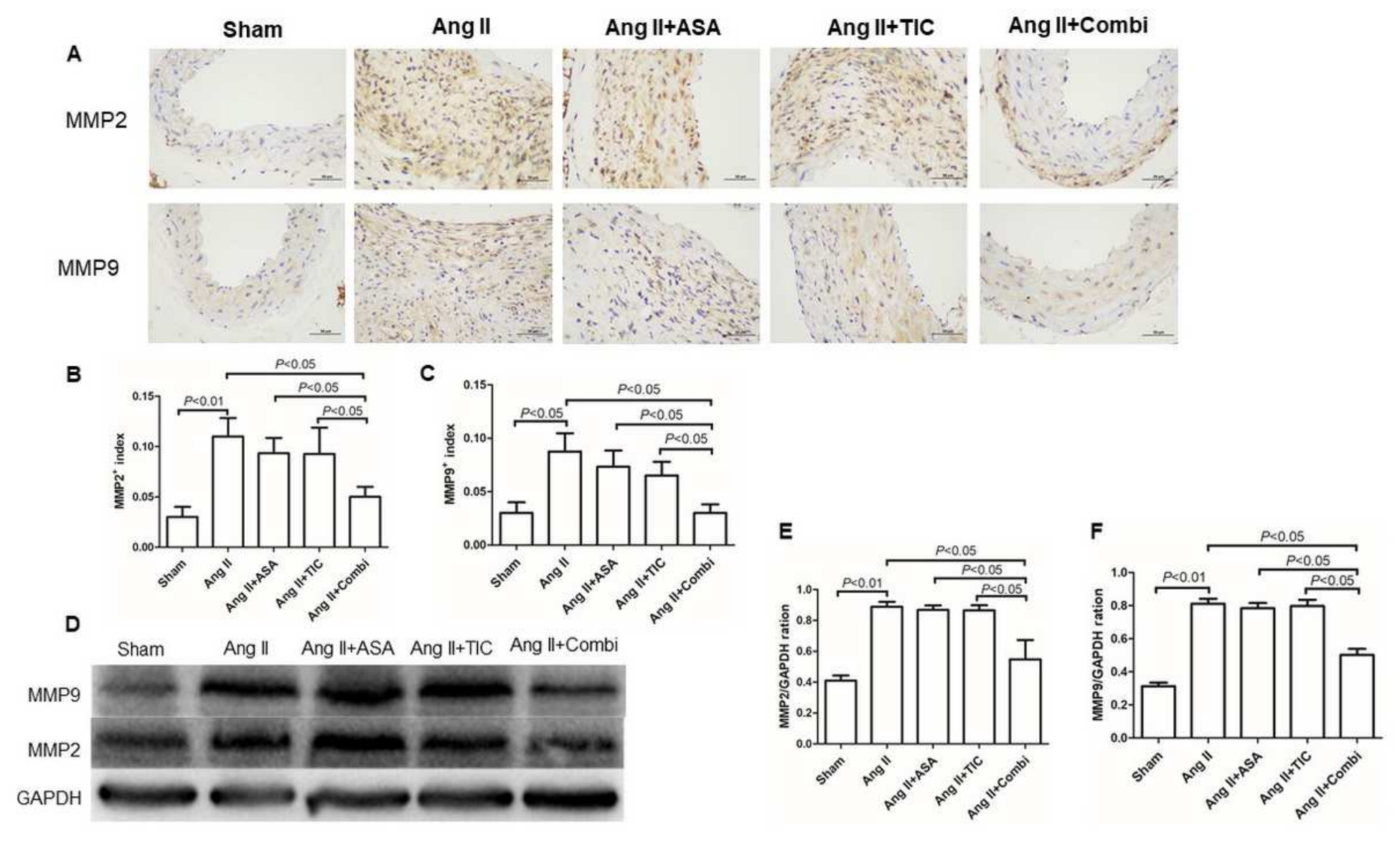

Figure 6

Combination therapy suppresses matrix metalloproteinases (MMPs) expression at the angiotensin II (Ang II)-induced abdominal aortic aneurysm (AAA) in ApoE-/- mice. A: Immunostaining for MMP-2 and MMP-9. B\&C: Quantitative analysis of MMP-2 and MMP-9, respectively. D: Representative Western blot band of MMP-2 and MMP-9. E\&F: Western blot analysis of MMP-2 and MMP-9. Abbreviations: ASA, aspirin; TIC, ticagrelor; Combi, combination.

Data are presented as mean $\pm S D ; n=5$ per group. 